\title{
On the Implied Volatility Layers Under the Future Risk-Free Rate Uncertainty *
}

\author{
Lin-Ye Hin $^{\dagger}$ AND Nikolai Dokuchaev ${ }^{\ddagger}$
}

Submitted: 2nd Jul 2013. Revised: 29th April 2014.

\begin{abstract}
This paper suggests a method of estimation of the implied volatility smile uncertainty of the observed options prices due to future risk-free rate uncertainty. The purpose is to quantify the range of uncertainty under different scenarios. We consider the setting where both the implied volatility and the risk free rate are calculated jointly from the observed option prices. Due to the cumulative risk-free rate uncertainty, the corresponding system of equations is underdetermined, leading to uncertainty in the volatility surface. We estimate the size of implied volatility layers between the surfaces representing the upper and lower bounds for the implied volatilities for the future risk-free rate uncertainty, defined by current Libor rate and the size of fluctuation estimated from the historical data.
\end{abstract}

Keywords: implied volatility surfaces, implied volatility layers, risk-free rate term structure, interest rate forecasting.

JEL codes: C14, C51, C58, C61, G13

*This work was supported by ARC grant of Australia DP120100928. In addition, we acknowledge provision of ICTsupport and computing resources by Curtin IT Services http://cits.curtin.edu.au. Curtin Information Technology Services (CITS) provides Information and Communication Technology systems and services in support of Curtin's teaching, learning, research and administrative activities. We acknowledge use of computing resources from the NeCTAR Research Cloud http://www.nectar.org.au. NeCTAR is an Australian Government project conducted as part of the Super Science initiative and financed by the Education Investment Fund.

${ }^{\dagger}$ Department of Mathematics and Statistics, Curtin University, GPO Box U1987, Perth, 6845 Western Australia.

¥Department of Mathematics and Statistics, Curtin University, GPO Box U1987, Perth, 6845 Western Australia.

This is a pre-copy-editing, author-produced PDF of an accepted for publication in International Journal of Financial Markets and Derivatives 3 (4), 392-408, following peer review. The definitive publisher-authenticated version is available online at http://www.inderscience.com. 


\section{Introduction}

This paper studies estimation of the uncertainty of the implied volatility surface (IVS), a manifold formed from the implied volatility parameter obtained by inverting the Black-Scholes pricing formula for a variety of observed options prices with different strikes and times to maturity. We investigate the uncertainty in calculating the implied volatility surface due to future risk-free rate uncertainty for a set of observed options prices. IVS is commonly used for pricing and risk management of exotic contingent claims.

In addition to the volatility, the Black-Scholes pricing formula includes the cumulate future risk-free interest rate. This parameter cannot be known with certainty, and has to be replaced by its forecast. For the purpose of calculating the implied volatility, an observed interest rate could be used as a proxy of the risk-free rate (Brenner and Subrahmanyam, 1988; Li, 2005; Haug, 2006, for example). The study of IVS dynamics together with the joint dynamics of the price and the current risk-free rate has been reported in voluminous extant literature such as Avellaneda, Friedman, Holmes and Samperi (1996), Cont and da Fonseca (2002), Cont, da Fonseca and Durrleman (2002), Goncalves and Guidolin (2004), Panigirtzoglou and Skiadopoulos (2004), Fengler, Härdle and Mammen (2007), Homescu (2011) and Câmara, Krehbiel and Li (2011) to name but a few. However, the observer risk-free rate does not necessary coincides with the future risk-free rate; there is always some uncertainty in the future dynamic of the risk-free rate. In the present paper, we focus on the study of the effect of the future risk-free rate uncertainty on the implied volatility surface. This issue was not address before.

We consider the setting where both the implied volatility and the risk-free rate are calculated from observed option prices representing the collective view of the market participants on the value of an option as well as the extent it should be discounted to give its net present value as the observed option price. We presume that since the instantaneous risk-free rate and instantaneous volatility are unknown between now and maturity, the observed option price implicitly embeds the expectation of the market participants on the average instantaneous risk-free rate and the average 
instantaneous volatility spanning the time to maturity that express themselves as the risk-free rate and implied volatility respectively.

We apply the so called ex-post approach, see, e.g., Singleton (1981) and Hedge (1990). For our model, this means using option prices observed at one spot time while considering risk-free rates observed at two different times, one observed at the spot time while the other observed at some subsequent time. The range between the risk-free rates observed at these two points in time is regarded as the range of future risk-free rate uncertainty across that period of time.

The model considered in the present paper is a modification of the model from Dokuchaev (2006), where it was suggested to calculate the pairs of implied parameters for pairs of option prices; the solution of the system of equations in that setting was unique, and the presence of an additional unknown parameter did not lead to uncertainty. However, that approach requires assigning the same volatility to different strike prices; this was inconsistent with the common approach to IVS. In the present paper, we want to remove this feature; our goal is to construct a volatility surface where vanilla options maturing at different dates and struck at different prices can be assigned its own volatility.

We consider a model where, for every available time to maturity, some observed option prices with different strikes are available. Each of these option prices has a value of implied volatility mapped to the time to maturity and strike price that characterises that option contract for a given spot price. Assuming that risk-free rate depends on time to maturity, options expiring on the same day will have one common risk-free rate. This leads to an underdetermined system of equations and uncertainty in the volatility surface. For any time to maturity, the system is underdetermined by one and there exists a set of possible solutions. For a given plausible range of uncertainty of the risk-free rate, we estimate the corresponding uncertainty in the implied volatility via the parametric dependence of the implied volatility surface on risk-free rate. We illustrate quantification of this range of uncertainty under different scenarios using historical data.

In our experiments, we study the implied volatility calculated for the S\&P500 options data. 
We used the realized fluctuation in the London Interbank Offer Rate (Libor) beyond the spot time as a plausible range of future risk-free rate uncertainty. These fluctuations were relatively small. However, it appears that the uncertainty that matches these fluctuations leads to a noticeable range of uncertainty for IVS. We used only few real life samples of Libor rather than the statistical characteristics of Libor as an illustration; in depth study on Libor time series can be found in, for example, Krehbiel and Adkins (2008) and Lu and Wu (2009).

The rest of the paper is organized as follows. In Section 2, we describe extended Black-Scholes framework to accommodate the notion of implied volatility and risk-free rate is outlined. In Section 3, we propose the framework to solve for implied volatilities and implied risk-free rate term structure from a system of nonlinear equations pertaining to a discrete ensemble of observed option prices. In Section 4, the uncertainty of implied volatility smile, given a range of uncertainty of implied risk-free rate, is illustrated using historical S\&P500 European vanilla call options data and the Libor term structure time series. In Section 5, the stochastic dynamics of the range of uncertainty for a set of options with the same time-to-maturity but different strike prices is examined. In Section 6, potential application and extension of this methodology is discussed.

\section{A review of Black-Scholes framework}

The celebrated Black-Scholes option pricing model (Black and Scholes, 1973) gives the option prices for the market model consisting of a risk free bond or bank account with the price $B(t), t \geq 0$, and a risky stock with price $S(t), t \geq 0$ where the prices of the stocks evolve as

$$
d S(t)=S(t)(a(t) d t+\delta(t) d W(t)), \quad t>0,
$$

where $W(t)$ is a Wiener process, $a(t)$ is an appreciation rate, and $\delta(t)$ is a random volatility coefficient. The initial price $S(0)>0$ is a given deterministic constant. The price of the bond evolves as

$$
B(t)=\exp \left(\int_{0}^{t} r(s) d s\right) B(0)
$$

where $r(t) \geq 0$ is a random process and $B(0)$ is given. 
It is assumed that $W(\cdot)$ is a standard Wiener process on a given standard probability space $(\Omega, \mathcal{F}, \mathrm{P})$, where $\Omega$ is a set of elementary events, $\mathcal{F}$ is a complete $\delta$-algebra of events, and $\mathrm{P}$ is a probability measure. Let $\mathcal{F}_{t}$ be a filtration generated by the currently observable data. We assume that the process $(S(t), a(t), \delta(t))$ is $\mathcal{F}_{t}$-adapted and that $\mathcal{F}_{t}$ does not depend on $\left\{W\left(t_{2}\right)-W\left(t_{1}\right)\right\}_{t_{2} \geq t_{2} \geq t}$. In particular, this means that the process $(S(t), a(t), \delta(t))$ is currently observable and $\delta(t)$ does not depend on $\left\{W\left(t_{2}\right)-W\left(t_{1}\right)\right\}_{t_{2} \geq t_{2} \geq t}$. Assume that $\mathcal{F}_{0}$ is the $P$-augmentation of the set $\{\emptyset, \Omega\}$, and that $a(t)$ does not depend on $\left\{W\left(t_{2}\right)-W\left(t_{1}\right)\right\}_{t_{2} \geq t_{2} \geq t}$. For simplicity, $a(t)$ is assumed to be a bounded process.

In the classical Black-Scholes framework, the standard implied volatility definition gives its value as a function of the risk-free interest rate, the option price, the strike price, the spot asset price, and time to maturity $\tau$. The standard definition of the implied volatility ignores the fact that, in reality, the risk-free rate is unknown and evolves dynamically over time. Therefore, the standard implied volatility at spot time $t$ is a conditional one and it depends on the future curve $\left.r(s)\right|_{s \in[t, T]}$. In fact, the Black-Scholes price at time $t$ depends only on the volatility process and on $\rho(t)=$ $\frac{1}{T-t} \int_{t}^{T} r(s) d s$, or on a single parameter of this curve, even if $r(\cdot)$ is random and depends on $(S, \sigma, W)$ where $W$ is the driving Wiener process. It is suggested to calculate the pair $\left(\sigma_{\text {imp }}(t), \rho_{\text {imp }}(t)\right)$ of two unconditionally implied parameters, where $\sigma_{\text {imp }}(t)$ is the unconditionally implied volatility, and $\rho_{\text {imp }}(t)$ is the unconditionally implied value of $r(t)$.

Consider the case of a European vanilla call with the payoff function $\max (S(T)-K, 0)$. Let the strike price $K>0$ be given and let $\delta(t)=\delta, r(t)=r$ be nonrandom and constant. Let $T>0$ be fixed and let $\tau=T-t$. Let $C_{B S}(\tau, S(t), \delta, r, K)$ denote the Black-Scholes price for the European vanilla call option defined as

$$
C_{B S}(\tau, S(t), \delta, r, K)=S(t) \Phi\left(d_{+}(\tau, S(t), \delta, r, K)\right)-K e^{-r \tau} \Phi\left(d_{-}(\tau, S(t), \delta, r, K)\right),
$$

where

$$
\begin{gathered}
d_{+}(\tau, S(t), \delta, r, K) \stackrel{\Delta}{=}\{\log (S(t) / K)+r \tau\} /(\delta \sqrt{\tau})+(\delta \sqrt{\tau}) / 2, \\
d_{-}(\tau, S(t), \delta, r, K) \stackrel{\Delta}{=} d_{+}(\tau, S(t), \delta, r, K)-\delta \sqrt{\tau}
\end{gathered}
$$

and $\Phi(d) \triangleq \frac{1}{\sqrt{2 \pi}} \int_{-\infty}^{d} e^{-\frac{s^{2}}{2}} d s$ 
Assume that $r(t)=r$ is constant. In practice, $\delta$ is often estimated from the inversion of the Black-Scholes formula, i.e., as a solution of the nonlinear equation

$$
C_{B S}(\tau, S(t), \delta, r, K)=\text { The observed price of option } .
$$

The solution for this equation, the nonrandom value $\delta_{\text {imp }}(t)$, is said to be the implied volatility. This implied volatility is not specifically related to a market model; it is used as a model-free characteristic to describe market uncertainty. Let

$$
\sigma(t) \triangleq\left(\frac{1}{T-t} \int_{t}^{T} \delta(s)^{2} d s\right)^{1 / 2}, \quad \text { and } \quad \rho(t) \triangleq \frac{1}{T-t} \int_{t}^{T} r(s) d s .
$$

If $\delta(\cdot)$ and $r(\cdot)$ are random but $\sigma(t)$ and $\rho(t)$ are not random at time $t$, the Black-Scholes formula (3) with $(\delta, r)$ replaced by $(\sigma(t), \rho(t))$ still gives the fair price of a European vanilla call option at time $t$, where where the fair price is understood in the sense of risk neutral pricing (see. e.g. Dokuchaev (2006)). Therefore, a given set of market option prices for different times to maturity gives an opportunity to calculate implied values $\sigma_{i m p}(t)$ and $\rho_{\text {imp }}(t)$,

\section{Larger sets of implied parameters}

The existing literature does not consider simultaneously both risk-free rate and implied volatility. Instead, risk-free rate is assumed to be known and deterministic. To fix notations for subsequent discussions, assume at the spot time $t$ for some strike price $K$ and some time to maturity $\tau$, we observe the price of an option $C=C(K, \tau)$. For simplicity of exposition, we consider European vanilla call prices only. If $\rho(t)$ is known and fixed, then the implied volatility surface represents a one-to-one mapping from $(K, \tau)$ to the option price expressed in terms of implied volatility $\sigma_{\text {imp }}(t)$ within the Black-Scholes framework. This is because the implied volatility is the only unknown parameter in the Black-Scholes European vanilla call price formula when the risk-free rate is known and fixed since the remaining parameters involved in the formula, spot asset price $S(t)$, strike price $K$ and time to maturity $\tau$, are readily observed from the market this option is traded on. The existence of a one-to-one bijective relation between the implied volatility and the option price enables the market participants to use implied volatility and option price interchangeably when 
referring to the value of a European vanilla call price, using the Black-Scholes formula as a tool for converting between these two quoting conventions. This practice of options value quotation using implied volatility in lieu of price is so widespread that even the Chicago Board of Options Exchange (CBOE) calculated the volatility index (VXO), now a de facto benchmark for stock market volatility, from 1993 to 2003 based on the implied volatility of S\&P100 index options data (Carr and $\mathrm{Wu}, 2006)$. In practice, it is expected that options contract with different $(K, \tau)$ characteristics matches it own implied volatility. Therefore, in the face of a discrete ensemble of $N$ observed option prices across $N$ unique $(K, \tau)$ pairs, the option prices can be represented by $N$ implied volatilities by solving a system of $N$ nonlinear equations where the uniqueness of solution is guaranteed.

If $\rho(t)$ is not known and fixed, the situation is different. Let $C\left(K_{i}, \tau_{j}\right), i=1, \ldots, n_{j}, j=$ $1, \ldots, m, N=\sum_{j=1}^{m} n_{j}$ be a discrete ensemble of $N$ observed vanilla call prices written on the same underlying asset observed at the same time where $m$ is the number of different times to maturity and $n_{j}$ is the number of options with time to maturity $\tau_{j}$ and let the corresponding Black-Scholes call prices incorporating risk-free rate and implied volatility be $C_{B S}\left(\tau_{j}, S(t), \sigma_{i m p, i, j}(t), \rho_{j}(t), K_{i}\right)$. The joint consideration of the risk-free rate $\rho_{j}(t)$ and the implied volatility $\sigma_{i m p, i, j}(t)$ is the solution to the system of $N$ equations

$$
C_{B S}\left(\tau_{j}, S(t), \sigma_{i m p, i, j}(t), \rho_{j}(t), K_{i}\right)=C\left(K_{i}, \tau_{j}\right), \quad i=1, \ldots, n_{j}, j=1, \ldots, m
$$

In our model, we assume that the risk-free rate depends on time to maturity through (4) and that options prices with the same time to maturity share the same risk-free rate. This leads to a model where the solution of $N$ observed call prices across $m$ unique times to maturity necessitates $N+m$ parameters to be estimated, namely $\sigma_{i m p, i, j}(t)$ and $\rho_{j}(t)$, where $i=1, \ldots, n_{j}, j=1, \ldots, m$.

When joint solution of the risk-free rates and and implied volatilities is considered, the oneto-one mapping no longer exists because there are more unknown parameters than equations. Uncertainty in the risk-free rate leads to uncertainty in the implied volatility. We suggest to study this uncertainty in the implied volatility surface in terms of possible range of $\sigma_{\text {imp }}(t)$ given certain interval $\left(\rho_{\min }, \rho_{\max }\right)$ for possible risk-free rate $\rho(t)$. 
The interval of future risk-free rate uncertainty $\left(\rho_{\min }, \rho_{\max }\right)$ can be constructed in different ways to simulate different scenarios. In ex-post study of future risk-free rate uncertainty using historical data, the risk-free rates observed at two different times, $\rho\left(t_{1}\right)$ and $\rho\left(t_{2}\right), \forall t_{2}>t_{1}$, from the historical time series of interest rate term structure can be used to construct $\left(\rho_{\min }, \rho_{\max }\right)$ to study the realized implied volatility smile uncertainty across different time frames. In portfolio scenario simulation, the current risk-free rate $\rho\left(t_{\text {spot }}\right)$ and a simulated future risk-free rate $\rho\left(t_{\text {future }}\right)$ designed to model the evolution of risk-free rate across the time interval $\left[t_{\text {spot }}, t_{\text {future }}\right]$ can be used to construct $\left(\rho_{\min }, \rho_{\max }\right)$ to facilitate quantification of the corresponding IVS uncertainty for risk management purposes by incorporating the implied volatility uncertainty into scenario simulation framework such as that proposed by Jamshidian and Zhu (1997). In rare event simulation for stress-testing, the current risk-free rate $\rho\left(t_{\text {spot }}\right)$ and a simulated future risk-free rate that mimics sharp, abrupt spikes in risk-free rate such as those observed in the catastrophic events of 2007 credit crunch can be used to construct $\left(\rho_{\min }, \rho_{\max }\right)$ for quantifying the corresponding implied volatility uncertainty and estimating the magnitude of potential system shock under extreme event scenario.

\section{Case study with cross-sectional empirical data}

We intend to demonstrate the impact of a small fluctuation of future risk-free rate on implied volatility. At the time when the study was carried out, 2009 to 2013 was the most recent time interval within which daily fluctuation of Libor term structure is small. This interval is depicted in Figure 1. Further, we need a subinterval to proceed with the case studies. We therefore chosen May 2011 as it is a subinterval in the middle of the wider time interval from 2009 to 2013.

The connection between the range of uncertainty of the risk-free rate and that of the implied volatility is illustrated below using historical options data with different expiry dates. The May 5th 2011 day-close data of the S\&P500 index European call options traded on the CBOE is used as an empirical example to illustrate the impact of a given interval of risk-free rate uncertainty on the range of the implied volatility uncertainty across strikes. 
For ex-post numerical demonstration, the range of risk-free rate uncertainty that mimics future risk-free rate uncertainty is inferred from historical time series of the Libor term structure downloaded from the Economic Research Division, Federal Reserve Bank of St. Louis. Table 1 depicts the interpolated Libor rates for this term structure on the dates used in this empirical illustration.

Table 1: Libor rates for the times to maturity corresponding to the options in the S\&P500 European call options on May 5th 2011, May 13th 2011 and May 27th 2011 respectively. The initial time to maturity refers to the time interval between May 5th 2011 and the expiry date of the options tabulated in each row. On May 13th 2011 and May 27th 2011, the times to maturity for the options are reduced by 8 and 22 days respectively.

\begin{tabular}{ccccc}
\hline Option expiry date & Initial time to maturity & \multicolumn{3}{c}{ Libor rate (decimal) } \\
\cline { 3 - 5 } (Y-M-D) & (day) & $2011-05-05$ & $2011-05-13$ & $2011-05-27$ \\
\hline $2011-06-18$ & 44 & 0.002133 & 0.001968 & 0.001728 \\
$2011-06-30$ & 56 & 0.002296 & 0.002121 & 0.001881 \\
$2011-07-16$ & 72 & 0.003014 & 0.002791 & 0.002522 \\
$2011-08-20$ & 107 & 0.003451 & 0.003205 & 0.002904 \\
$2011-09-17$ & 135 & 0.003666 & 0.003412 & 0.003096 \\
$2011-09-30$ & 148 & 0.005030 & 0.004759 & 0.004399 \\
$2011-12-17$ & 226 & 0.005261 & 0.004990 & 0.004630 \\
$2011-12-30$ & 239 & 0.006644 & 0.006365 & 0.006022 \\
$2012-03-17$ & 317 & 0.006876 & 0.006596 & 0.006254 \\
$2012-03-30$ & 330 & 0.007520 & 0.007380 & 0.007295 \\
$2012-06-16$ & 408 & 0.007520 & 0.007380 & 0.007295 \\
$2012-12-22$ & 597 & 0.007520 & 0.007380 & 0.007295 \\
$2013-12-21$ & 961 & \multicolumn{3}{c}{} \\
\hline
\end{tabular}

Albeit the range of future risk-free rate uncertainty considered in the current numerical demonstration based on historical data across a short period of time in May 2011 is small, this approach can potentially accommodate wider ranges of future risk-free rate uncertainty in the context of scenario simulation and extreme event stress test. Figure 1 depicts the contrasting trends of Libor term structure across time during different market condition. The upper panel in this figure illustrates the sharp fluctuation of interest rate that can take place within a short period of time in tumulus market conditions. In this context the range of future risk-free rate uncertainty $\left(\rho_{\min }, \rho_{\max }\right)$ can be large. On the contrary, $\left(\rho_{\min }, \rho_{\max }\right)$ is smaller during relatively calm market condition as depicted in the lower panel. 
We compare the impact of future risk-free rate uncertainty across the same time interval on options expiring on different dates. Two subsets of data corresponding to options expiring on September 30th 2011 and March 30th 2012 respectively are used to contrast the implied volatility uncertainty in the presence of risk-free rate uncertainty across the same period of time for options with different expiry dates. The risk-free rate at spot time is inferred from the Libor term structure observed May 5th 2011 while the future risk-free rate is inferred from the Libor term structure observed on May 13th 2011. These rates are used to form the future risk-free rate uncertainty range $\left(\rho_{\min }, \rho_{\max }\right)$ to calculate the corresponding implied volatility uncertainty. For options expiring on September 30th 2011, $\rho_{\min }=0.003412$ and $\rho_{\max }=0.003666$; whereas for options expiring on March 30th 2012, $\rho_{\min }=0.006596$ and $\rho_{\max }=0.006876$. These profiles, depicted in Figure 2 and Table 2, demonstrates that the region of the implied volatility smile deep into the money exhibits a wider range of uncertainty than out of the money region. This feature is more pronounced for longer dated options.

In addition, the impact of future risk-free rate uncertainty across different time intervals ahead of the spot time on options with the same expiry date is examined. Implied volatility uncertainty in the presence of risk-free rate uncertainty across two different time intervals based on options expiring on March 30th 2012 are depicted in two separate panels in Figure 3 as well as Table 2 to contrast the corresponding range of implied volatility uncertainty. The shorter time interval mimics future risk-free rate uncertainty between the spot time, May 5th 2011, and a subsequent date, May 13th 2011, where $\rho_{\min }=0.003412$ and $\rho_{\max }=0.003666$ are inferred from the Libor term structures observed on these two dates. The longer time interval mimics future risk-free rate uncertainty between the spot time and a date further into the future, May 27th 2011, where $\rho_{\min }=0.003412$ and $\rho_{\max }=0.003666$ are inferred from the Libor term structures observed on the corresponding dates. The region of uncertainty for the implied volatility is larger for scenario mimicking future risk-free rate uncertainty across a longer time interval due to a wider range of risk-free rate uncertainty. This is a data dependent phenomenon as the evolution of interest rate across time is non-monotonic and can be such that the interval between spot rate and the rate more distant into the future may be narrower than that between the spot rate and an interest rate at a time 
in the nearer future. Suffice to note that the range of risk-free rate uncertainty between the spot time and any two different time points ahead will be different.

The implied volatility for these two subsets of options data calculated assuming the risk-free rate to be the Libor rates observed on May 5th, 13th or 27th 2011 are tabulated in Table 2 for a range of near the money options. The spot price was 1335.1. The same range of risk-free rate uncertainty results in a wider range of implied volatility uncertainty for into the money options and long dated options as opposed to those otherwise. The range of implied volatility uncertainty is wider for longer dated options.

Table 2: Implied volatility for near the money S\&P500 European call options observed on May 5th 2011 calculated assuming the risk-free rate $\rho$ to be the Libor rates observed on May 5th, 13th or 27th 2011, which are $0.003666,0.003412 \& 0.003096$, and $0.006876,0.006596 \& 0.006254$ for options maturing on September 30th 2011 and March 30th 2012 respectively.

\begin{tabular}{|c|c|c|c|c|c|c|}
\hline \multirow[t]{2}{*}{$\begin{array}{l}\text { Strike } \\
\text { price }\end{array}$} & \multicolumn{3}{|c|}{$\begin{array}{c}\text { Implied volatility for option } \\
\text { that expire on 2011-09-30 } \\
\text { assuming } \rho \text { as Libor rate observed on }\end{array}$} & \multicolumn{3}{|c|}{$\begin{array}{c}\text { Implied volatility for option } \\
\text { that expire on 2012-03-30 } \\
\text { assuming } \rho \text { as Libor rate observed on }\end{array}$} \\
\hline & 2011-05-05 & 2011-05-013 & 2011-05-27 & 2011-05-05 & 2011-05-013 & $2011-05-27$ \\
\hline 1000 & 0.040102 & 0.040045 & 0.039976 & 0.105296 & 0.125681 & 0.136954 \\
\hline 1100 & 0.209278 & 0.210256 & 0.211474 & 0.186461 & 0.187436 & 0.188603 \\
\hline 1200 & 0.200485 & 0.200910 & 0.201416 & 0.184566 & 0.185122 & 0.185771 \\
\hline 1300 & 0.177711 & 0.177953 & 0.178254 & 0.173211 & 0.173606 & 0.174066 \\
\hline 1400 & 0.152565 & 0.152724 & 0.152885 & 0.158275 & 0.158538 & 0.158845 \\
\hline 1500 & 0.132203 & 0.132285 & 0.132386 & 0.143266 & 0.143439 & 0.143684 \\
\hline
\end{tabular}

\section{Case study with longitudinal empirical data}

In this section, we study the dynamics of the implied volatility uncertainty for a set of options across a number of consecutive trading days assuming a fixed range of future risk-free rate uncertainty throughout these trading days. Although in reality the future risk-free rate uncertainty changes from day to day, the study of the fluctuation of future risk-free rate uncertainty and the reduction of time to maturity on the implied volatility uncertainty is complicated and difficult to 
interpret. The current approach, albeit simple, allows us to concentrate on quantifying the trend of implied volatility uncertainty as the time to maturity of the options decreases conditional on the same range of risk-free rate uncertainty. Historical data of S\&P500 index European call options traded on CBOE from May 3rd 2011 to May 31st 2011 inclusive is used.

Two subsets of data corresponding to options expiring on September 30th 2011 and March 30th 2012 respectively are used to contrast the implied volatility uncertainty for a fixed range of risk-free rate uncertainty across the same period of time for options with different expiry dates. The risk-free rate at spot time is inferred from the Libor term structure observed May 5th 2011 while the future risk rate is inferred from the Libor term structure observed on May 31st 2011. For options expiring on September 30th 2011, $\rho_{\min }=0.003022$ and $\rho_{\max }=0.003740$. For longer-dated options expiring on December 21st 2013, $\rho_{\min }=0.007295$ and $\rho_{\max }=0.007578$.

The range of implied volatility uncertainty for every option contract is quantified with reference to the prices observed on each of the trading dates considered. Let $\Delta(t)=\sigma_{\max }(t)-\sigma_{\min }(t)$ denote this range while $t$ be the trading date. The time series of $\Delta(t)$ for these two sets of options are tabulated in Table 3 and depicted in Figure 4. For longer dated options, the range of implied volatility uncertainty for a given range of risk-free rate uncertainty is wider. As the remaining time to maturity for the option decreases, this range narrows. However, the decrease of $\Delta(t)$ across time is neither smooth nor monotonic. Instead, it exhibits stochastic behaviour across time, as shown in Figure 4. Additionally, given the same range of future risk-free rate uncertainty, the range of implied volatility uncertainty for longer dated options is wider. These observations are due to the fact that the prices of options with the same expiry date but lower strike prices, and that the prices of options with the same strike price but more distant expiry date are in general higher than their counterparts and as such entails a larger magnitude of $\Delta(t)$. This demonstrates that the uncertainty of implied volatility due to the risk-free rate uncertainty has a more pronounced impact on pricing path-dependent contingent claims that depends heavily on the long dated region of the implied volatility surface.

One of the primary impetus for profiling the magnitude of the volatility uncertainty range for 
Table 3: Implied volatility uncertainty, $\Delta(t)$, for near the money S\&P500 European call options observed for each trading day in May 2011 calculated assuming the risk-free rate $\rho$ to be the Libor rates observed on May 2nd or 27th 2011, which are $0.003022 \& 0.003740$, and $0.007295 \&$ 0.007578 for options maturing on September 30th 2011 and December 21st 2013 respectively.

\begin{tabular}{|c|c|c|c|c|c|}
\hline \multicolumn{3}{|c|}{ Options expiring on 2011-09-30 } & \multicolumn{3}{|c|}{ Options expiring on $2012-03-30$} \\
\hline \multirow{2}{*}{$\begin{array}{c}\text { Remaining } \\
\text { days to maturity }\end{array}$} & \multicolumn{2}{|c|}{$\Delta(t) \times 10^{4}$ for strike } & \multirow{2}{*}{$\begin{array}{c}\text { Remaining } \\
\text { days to maturity }\end{array}$} & \multicolumn{2}{|c|}{$\Delta(t) \times 10^{4}$ for strike } \\
\hline & 1300 & 1350 & & 1300 & 1350 \\
\hline 150 & 8.1109 & 6.1961 & 963 & 15.2900 & 13.6729 \\
\hline 149 & 7.2671 & 5.5069 & 962 & 14.7074 & 13.1785 \\
\hline 148 & 6.8184 & 4.8442 & 961 & 14.3815 & 13.0682 \\
\hline 147 & 7.2423 & 5.2465 & 960 & 14.7226 & 13.2310 \\
\hline 144 & 7.2388 & 5.1645 & 957 & 14.8460 & 13.5592 \\
\hline 143 & 7.6903 & 5.4952 & 956 & 15.2220 & 13.4902 \\
\hline 142 & 7.0732 & 4.8597 & 955 & 14.7675 & 13.0340 \\
\hline 141 & 7.4327 & 5.1182 & 954 & 14.7660 & 13.2549 \\
\hline 140 & 6.9275 & 5.1113 & 953 & 14.2565 & 13.2757 \\
\hline 137 & 6.0202 & 4.5480 & 950 & 14.2170 & 12.7400 \\
\hline 136 & 6.1249 & 4.9204 & 949 & 14.2015 & 13.0187 \\
\hline 135 & 6.9528 & 4.9476 & 948 & 14.3012 & 12.8745 \\
\hline 134 & 7.0739 & 5.4153 & 947 & 14.4494 & 13.1024 \\
\hline 133 & 6.8918 & 4.5891 & 946 & 14.4809 & 12.5228 \\
\hline 130 & 5.6973 & 4.1463 & 943 & 14.0775 & 12.4794 \\
\hline 129 & 5.6160 & 4.1096 & 942 & 13.4644 & 12.4921 \\
\hline 128 & 6.0966 & 4.2712 & 941 & 14.0185 & 12.7519 \\
\hline 127 & 6.0172 & 4.6379 & 940 & 14.1189 & 12.7614 \\
\hline 126 & 6.3804 & 4.3716 & 939 & 14.0613 & 12.5497 \\
\hline 122 & 6.9529 & 4.9584 & 935 & 14.3995 & 13.1295 \\
\hline
\end{tabular}


options with different strike and time to maturity characteristics across a consecutive sequence of trading days is to investigate the dynamics of this process. In particular, Table 3 and Figure 4 demonstrates that the process is not smooth, exhibiting dynamics that is stochastic in nature resembling the dynamics of implied volatility itself.

\section{Conclusions}

The current work proposes joint inference of the implied volatility and risk-free rate from a set of observed options prices as solution to an underdetermined system of nonlinear equations. The joint consideration of both parameters admits multiple solutions, leading to implied parameter uncertainty. The quantifying of the implied volatility uncertainty due to the future risk-free rate uncertainty can help to improve option pricing and general risk assessment for financial markets.

The investigation pertaining to the future risk-free rate uncertainty undertaken in this paper fits into the strand of literature that seek to describe the fluctuations of interest rates for risk management and trading strategies optimization in the fixed income market (see Kanevski, Maignan, Pozdnoukhov and Timonin, 2008; Cont, 2005; Jamshidian and Zhu, 1997, for example). For the purpose of risk management, short term interest rate fluctuation is the subject of interest. While Aït-Sahalia (1996) has studied the evolution of spot interest rate based on seven day Eurodollar deposit interest rates between 1973 and 1995 to model interest rate evolution, we investigated instead, in this paper, the ex post impact of future risk-free rate fluctuation one to three weeks ahead of the spot time on implied volatility. Despite the difference in the time frames considered, the stochastic evolution of interest rate and the increased daily fluctuation of interest rate during volatile market conditions are noticeable in both Figure 1 of Aït-Sahalia (1996) and Figure 1 of this paper.

The historical time series of the Libor term structure for the time frame corresponding to calm market conditions between 2009 and 2013 depicted in Figure 1 and tabulated in Table 1 for May 2011 demonstrate that during this time frame, the daily functuation of the Libor term structure tend to demonstrate a dominant picture of parallel shift of interest rates spanning the entire tenor, 
a feature of interest rate dynamic evolution reported by Bouchaud, Sagna, Cont, El-Karoui and Potters (1999), and Litterman and Scheinkman (1991), among others. In contrast, for the time frame when the market is volatile such as that between December 2008 and April 2009, the daily fluctuation of the Libor term structure tend to demonstrate a dominant picture of opposite changes in short and long term rates such that the interest rates at shorter end of the term structure increase while the interest rates at longer end of the term structure decrease, another feature of interest rate dynamic evolution reported by Bouchaud et al. (1999). While the current work investigated the impact of risk-free rate uncertainty on implied volatility in an underdefined system of nonlinear equations, Hin and Dokuchaev (2014) investigated this impact in a different framework that lead to an overdefined system of equation. Some related bibliography can be found in Butler and Schachter (1996), who suggested using a similar system for calculations of implied volatility distributions for the case of option prices obtained via the unbiased estimate of option price for random volatility.

We observed that the dependence of the layers for the IVS on the time to maturity is not smooth. It could be useful to consider smoothing of the implied volatility layers using splines, in the framework of Fengler and Hin (2013). It could be also interesting to compare the correlations of the implied volatility extracted from the volatility surface described above with the historical volatilities calculated by different methods such as suggested in Dokuchaev (2014). We leave this for future research. 


\section{References}

Aït-Sahalia, Y. (1996). Nonparametric pricing of interest rate derivative securities, Econometrica 64(3): $527-560$.

Avellaneda, M., Friedman, C., Holmes, R. and Samperi, D. (1996). Calibrating volatility surfaces via relative-entropy minimization. Working paper. Available at SSRN: http://ssrn.com/abstract=648 or http://dx.doi.org/10.2139/ssrn.648.

Black, F. and Scholes, M. (1973). The pricing of options and corporate liabilities, Journal of Political Economy 81(3): 637-654.

Bouchaud, J.-P., Sagna, N., Cont, R., El-Karoui, N. and Potters, M. (1999). Phenomenology of the interest rate curve, Applied Mathematical Finance 6: 209-232.

Brenner, M. and Subrahmanyam, M. (1988). A simple formula to compute the implied standard deviation, Financial Analyst Journal (September-October): 80-83.

Butler, J. and Schachter, B. (1996). The statistical properties of parameters inferred from the black-scholes formula, International Review of Financial Analysis 3: 223-235.

Câmara, A., Krehbiel, T. and Li, W. (2011). Expected returns, risk premia, and volatility surfaces implicit in option market prices, Journal of Banking $\mathcal{F}$ Finance 35(1): 215 - 230.

Carr, P. and Wu, L. (2006). A tale of two indices, Journal of Derivatives (Spring): 13-29.

Cont, R. (2005). Modeling term structure dynamics: An infinite dimensional approach, International Journal of Theoretical and Applied Finance 8: 357-380.

Cont, R. and da Fonseca, J. (2002). The dynamics of implied volatility surfaces, Quantitative Finance 2(1): 45-60.

Cont, R., da Fonseca, J. and Durrleman, V. (2002). Stochastic models of implied volatility surfaces, Economic Notes 31(2): 361-377. 
Dokuchaev, N. (2006). Two unconditionally implied parameters and volatility smiles and skews, Applied Financial Economics Letters 2: 199-204.

Dokuchaev, N. (2014). Volatility estimation from short time series of stock prices, Journal of Nonparametric statistics 26(2): 373-384.

Fengler, M., Härdle, W. and Mammen, E. (2007). A semiparametric factor model for implied volatility surface dynamics, Journal of Financial Econometrics 5(2): 189-218.

Fengler, M. R. and Hin, L.-Y. (2013). Semi-nonparametric estimation of the call price surface under strike and time-to-expiry no-arbitrage constraints. Working paper. Available at SSRN: http://ssrn.com/abstract=1910737.

Goncalves, S. and Guidolin, M. (2004). Predictable dynamics in the S\&P500 index options implied volatility surface. EFMA 2003 Helsinki Meetings. Available at SSRN: http://ssrn.com/abstract=406697 or http://dx.doi.org/10.2139/ssrn.406697.

Haug, E. (2006). The complete Guide to Option Pricing Formulas (2nd ed.), McGraw-Hill, New York.

Hedge, S. (1990). An ex post valuation of the quality option implicit in the treasury bond futures contract, Journal of Banking and Finance 14(4): 741-760.

Hin, L. Y. and Dokuchaev, N. (2014). An analysis of volatility spread via the risk-free rate proxy. Working paper. Available at SSRN: http://ssrn.com/abstract=2400872.

Homescu, C. (2011). Implied volatility surface: Construction methodologies and characteristics. Available at SSRN: http://ssrn.com/abstract=1882567 or http://dx.doi.org/10.2139/ssrn.1882567.

Jamshidian, F. and Zhu, Y. (1997). Scenario simulation: Theory and methodology, Finance and Stochastics 1: 43-67.

Kanevski, M., Maignan, M., Pozdnoukhov, A. and Timonin, V. (2008). Interest rates mapping, Physica A 387: 3897-3903. 
Krehbiel, T. and Adkins, L. (2008). Extreme daily changes in U.S. Dollar london inter-bank offer rates, International Review of Economics and Finance 17(3): 397-411.

Li, S. (2005). A new formula for computing implied volatility, Applied Mathematics and Computation 170(1): 611-625.

Litterman, R. and Scheinkman, J. (1991). Common factors affecting bond returns, Journal of Fixed Income 1(June): 54-61.

Lu, B. and Wu, L. (2009). Macroeconomic releases and the interest rate term structure, Journal of Monetary Economics 56(6): 872-884.

Panigirtzoglou, N. and Skiadopoulos, G. (2004). A new approach to modeling the dynamics of implied distributions: Theory and evidence from the S\&P500 options, Journal of Banking and Finance 28: 1499-1520.

Singleton, K. (1981). Extracting measures of ex ante real interest rates from ex post rates: A comment, Carnegie-Rochester Conference Series on Public Policy 15: 201-212. 
Figure 1: Historical time series of the Libor term structure. The upper panel depicts the Libor term structure evolution from November 30th 2007 to July 16th 2009 during the credit crunch. The lower panel depicts the Libor term structure evolution from July 16th 2009 and May 31st 2013 during a relatively calm market period.
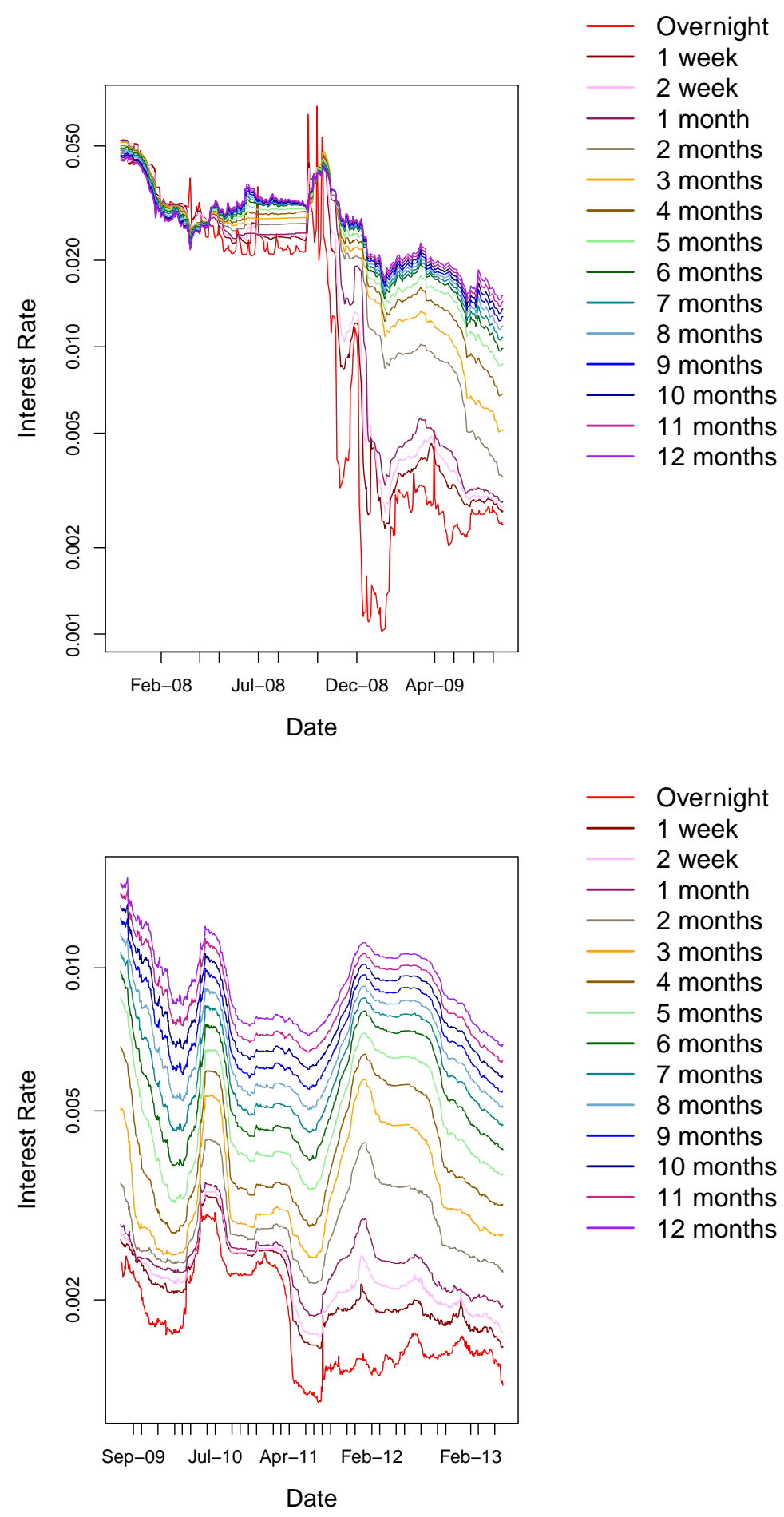
Figure 2: The regions of implied volatility uncertainty, highlighted as shaded areas, for a given range of risk-free rates uncertainty, estimated from historical Libor time series, are illustrated using SPX500 index European call options data. The Libor rates observed on May 5th and May 13th 2011 for options expiring on September 30th 2011 are 0.003666 and 0.003412 , while those for March 30th 2012 are 0.006876 and 0.006596 respectively.
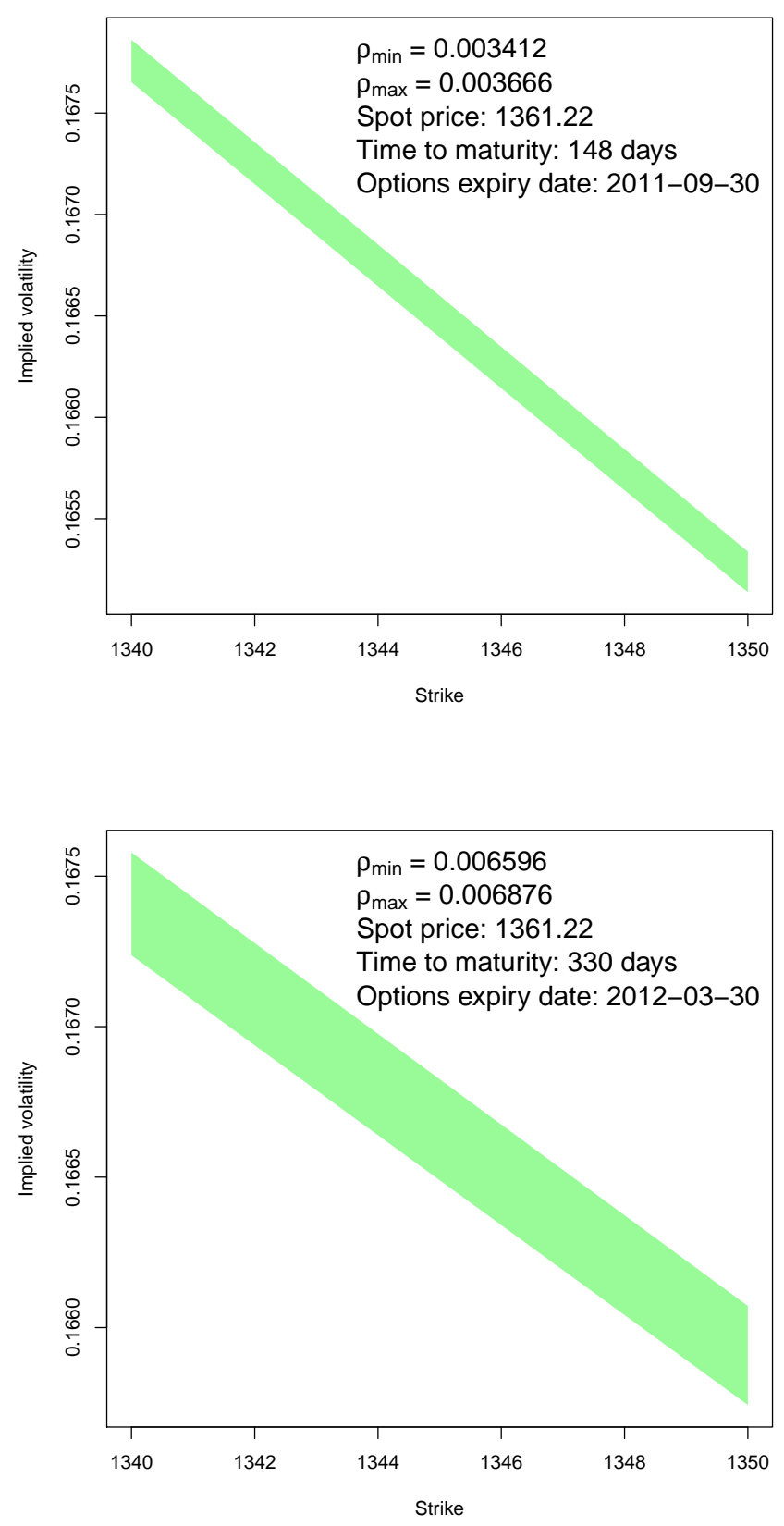
Figure 3: The regions of implied volatility uncertainty, highlighted as shaded areas, for different ranges of risk-free rates uncertainty, estimated from historical Libor time series, are illustrated using SPX500 index European call options data. For options expiring on March 30th 2012, the Libor rates observed on May 5th, May 13th and May 27th 2011 are 0.006876, 0.006596 and 0.006254 respectively.
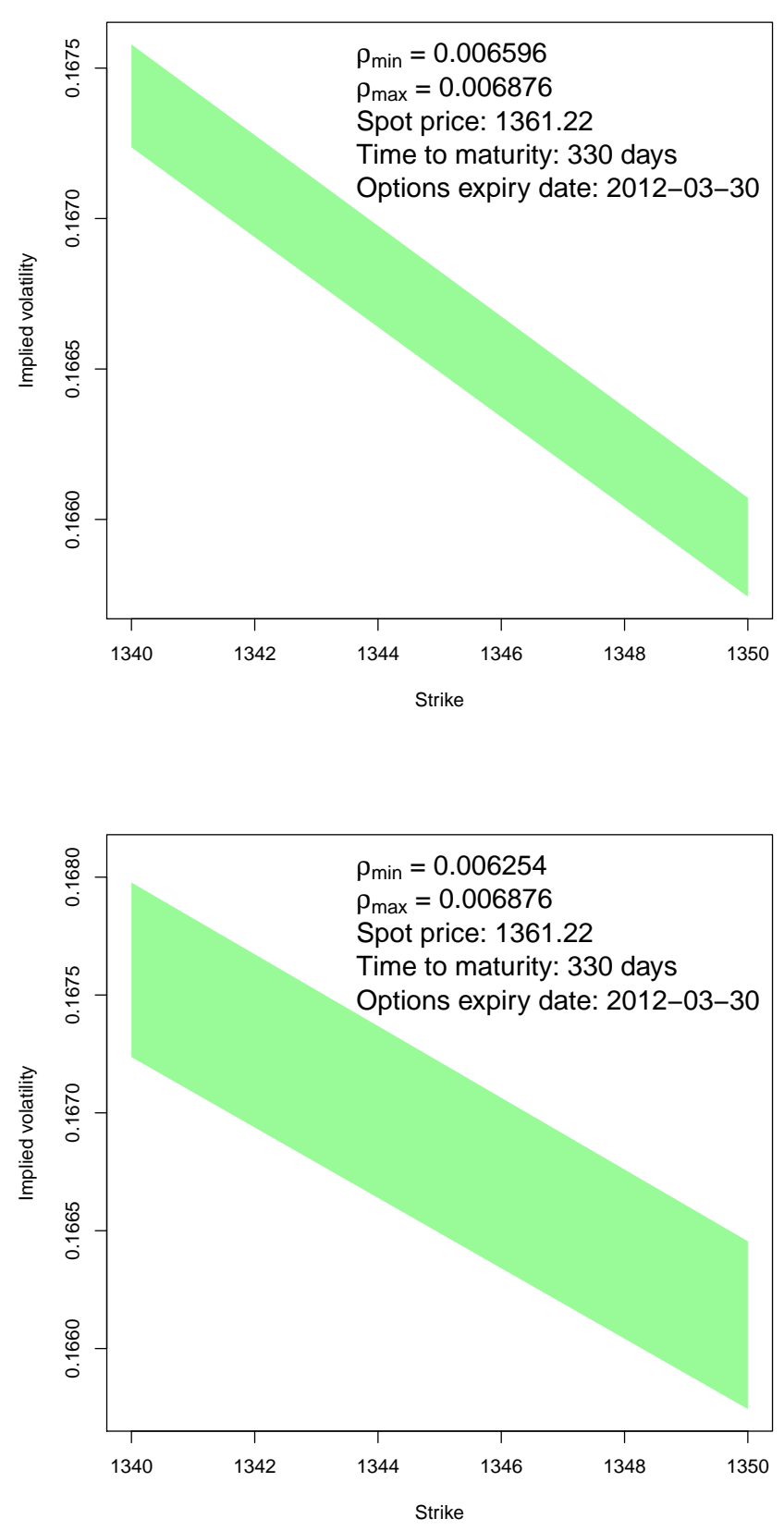
Figure 4: Dynamic evolution, from May 3rd to May 31st 2011, of the implied volatility uncertainty ranges for S\&P500 index European call options that expire on September 30th 2011 and December 21st 2013. The Libor rates observed on May 3rd and May 31st 2011 for options expiring on September 30th 2011 are 0.003740 and 0.003022, while those for December 21st 2013 are 0.007578 and 0.007295 respectively.
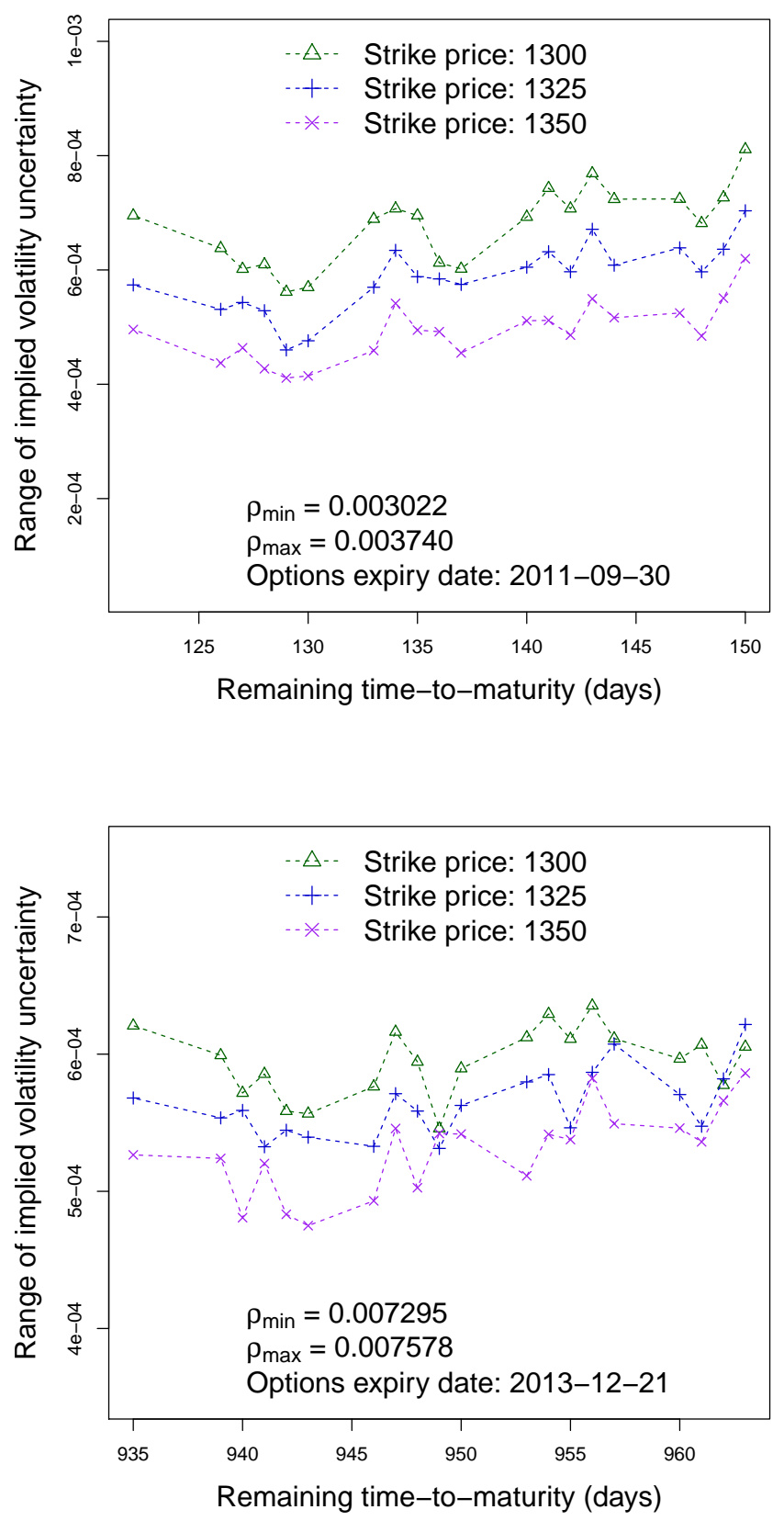